\title{
ZHX2 Interacts with Ephrin-B and Regulates Neural Progenitor Maintenance in the Developing Cerebral Cortex
}

\author{
Chen Wu, ${ }^{1,2}$ Runxiang Qiu, ${ }^{1}$ Jun Wang, ${ }^{1,2}$ Heying Zhang, ${ }^{1}$ Kiyohito Murai, ${ }^{1}$ and Qiang $\mathrm{Lu}^{1}$ \\ ${ }^{1}$ Department of Neurosciences, Beckman Research Institute of the City of Hope, and ${ }^{2}$ City of Hope Graduate School of Biological Sciences, Duarte, California \\ 91010
}

Neural progenitor cells in the ventricular zone of the developing mammalian cerebral cortex give rise to specialized cortical cell types via consecutive rounds of proliferation and differentiation, but the mechanisms by which progenitor cell self-renewal and differentiation are regulated during cortical development are not well understood. Here, we show that zinc-finger and homeodomain protein 2 (ZHX2) is specifically expressed in neural progenitor cells during cortical neurogenesis. ZHX2 binds to the cytoplasmic domain of ephrin-B1, which is expressed in cortical neural progenitors and plays a role in neural progenitor cell maintenance. ZHX2 acts as a transcriptional repressor in cell, and its repressor activity is enhanced by coexpression of an ephrin-B1 intracellular domain. Blocking ZHX2 function in cultured neural progenitor cells or in the embryonic cortex causes neuronal differentiation, whereas overexpression of ZHX2 and an ephrin-B1 intracellular domain disrupts the normal differentiation of cortical neural progenitor cells. This study identifies ZHX2 as a novel regulator of neural progenitor cell maintenance and suggests a potential nuclear mechanism of the ephrin-B function in the cortex.

\section{Introduction}

During the development of the mammalian cerebral cortex, neural progenitor cells located in the ventricular zone (VZ) generate the majority of projection neurons via consecutive rounds of expansion and differentiation (McConnell, 1995; Monuki and Walsh, 2001; Temple, 2001; Gupta et al., 2002; Rash and Grove, 2006; Bystron et al., 2008; Caviness et al., 2008), but the mechanisms by which progenitor self-renewal and differentiation are regulated during cortical development are not well understood (Fishell and Kriegstein, 2003; Götz and Huttner, 2005; Molyneaux et al., 2007; Noctor et al., 2007; Pontious et al., 2008). In a previous study, we found that ephrin-B1 plays a critical role in maintaining the neural progenitor state during cerebral cortical development (Qiu et al., 2008). Ephrin-B is the transmembrane ligand of the Eph receptor tyrosine kinases and transduces a "reverse signal" (signaling into the ephrin-B-expressing cells) in ephrin-Eph-mediated neural functions (Flanagan and Vanderhaeghen, 1998; Cowan and Henkemeyer, 2002; Drescher, 2002; Poliakov et al., 2004; Davy and Soriano, 2005; Egea and Klein, 2007; Arvanitis and Davy, 2008; Pasquale, 2008). Ephrin-B is thought to elicit reverse signaling as an anchored membrane protein through interaction with cytosolic proteins, such as PDZRGS3. It was recently reported that ephrin-B could be processed

Received Dec. 8, 2008; revised April 15, 2009; accepted May 1, 2009.

This work was supported by National Institutes of Health Grant NS052388 to Q.L. We thank Mariko Lee, Brian Armstrong, and Paul Salvaterra for helping with confocal imaging; Yanhong Shi and Barry Forman for helping with luciferase transcription assay; and Michael Barish, Paul Salvaterra, Toshifumi Tomoda, and Elizabeth Engle for thoughtful discussions, suggestions, and help. We thank John G. Flanagan, in whose laboratory ZHX2 was initially isolated in an ephrin-B two-hybrid screen. We thank the Developmental Studies Hybridoma Bank.

Correspondence should be addressed to Qiang Lu, Department of Neurosciences, Beckman Research Institute of the City of Hope, 1500 East Duarte Road, Duarte, CA 91010. E-mail: qlu@coh.org.

C. Wu's present address: Department of Neurology, Children's Hospital Boston, Boston, MA 02115

D01:10.1523/JNEUROSCI.5841-08.2009

Copyright $\odot 2009$ Society for Neuroscience $\quad$ 0270-6474/09/297404-09\$15.00/0 into an intracellular fragment both in the mouse brain and in transfected cells, likely via presenilin-dependent intramembrane proteolysis (Georgakopoulos et al., 2006; Tomita et al., 2006). This raised a possibility that ephrin-B, like Notch and many other type I transmembrane proteins, might interact with a nuclear factor to modulate gene expression using a free cytosolic fragment (Fortini, 2002; Selkoe and Kopan, 2003). We were, therefore, interested to search for a potential nuclear factor that could interact with the intracellular fragment of ephrin-B.

In a previous screen for interacting proteins of ephrin-B cytoplasmic domain (Lu et al., 2001), one of the positive cDNA clones identified encoded a fragment of zinc-finger and homeodomain protein 2 (ZHX2). ZHX2 is a member of the zinc-fingers and homeoboxes (ZHX) family which include ZHX1, ZHX2, and ZHX3 (Barthelemy et al., 1996; Kawata et al., 2003a,b). ZHX proteins form homodimers and heterodimers and act as transcription repressors in several cell types studied (Kawata et al., 2003a,b; Perincheri et al., 2005; Liu et al., 2006). The isolation of ZHX2 in an ephrin-B yeast two-hybrid screen suggested that ZHX2 could be a candidate nuclear factor for the ephrin-B intracellular fragment discovered in recent studies (Georgakopoulos et al., 2006; Tomita et al., 2006). In this study, we present evidence that ZHX2 and ephrin-B1 cytoplasmic domain can physically interact and regulate transcription. We further show that ZHX2 is also expressed in neural progenitor cells during cortical neurogenesis, and interfering with ZHX2 function or overexpression of ZHX2 leads to dysregulation in cortical neural progenitor maintenance and differentiation.

\section{Materials and Methods}

Plasmids and antibodies. Green fluorescent protein (GFP)-ephrinB1CD, hemagglutinin (HA)-ephrinB1CD, Myc6-Z0, Gal4-ZHX2, Gal4ZHX2-VP16 and DsRed-ZHX2 were cloned into pcDNA3.1Zeocin (Invitrogen). Flag-ZHX2-VP16 was cloned into pEF-Ub-GFP (modified 
from pEFGM) which carries an Ubiquitin promoter-enhanced GFP (EGFP) expression cassette for tracing. Primary antibodies used in this study included a rabbit polyclonal anti-ephrin-B antibody C18 (Santa Cruz Biotechnology), anti-doublecortin (DCX) (Santa Cruz Biotechnology), anti-bromodeoxyuridine (BrdU) (Sigma), Rat anti-HA (Roche), Sheep anti-digoxigenin-alkaline phosphatase (Roche), anti-GFP (Roche, Invitrogen). Anti-ZHX2 antibody was raised against peptide KPSEATSDRSEGS by Zymed. All secondary antibodies were purchased from Jackson ImmunoResearch Laboratories (Rhod Red-X-AffiniPure and Cy2 AffiniPure conjugated).

Cell culture and transfection. COS-7 cells and HEK293 cells were cultured in DMEM (Invitrogen) with 10\% bovine serum (Sigma). Transfection of COS-7 was done using Fugene6 (Roche) following manufacturer's instruction; transfection of HEK293 was done using calcium phosphate method.

Affinity binding studies and Western blot. Glutathione S-transferase (GST), GST fusion deletion mutant proteins, and His6-ZHX2 were expressed in bacteria strain BL21gold. The cell pellet was lysed in lysis buffer [25 mm Tris 7.5, $140 \mathrm{~mm} \mathrm{NaCl}, 1 \times$ protease inhibitors mixture (Roche), $1 \%$ Triton X-100], and the supernatant was loaded to glutathione agarose beads (Sigma) or Talon beads to prepare GST-ephrin-B or His6ZHX2 affinity beads. Full-length ZHX2 or Myc6-Z0 was expressed in HEK293 cells; $48 \mathrm{~h}$ after transfection, the cells were lysed in lysis buffer, and clear supernatant was loaded onto GST affinity beads for pull-down assay. For preparing endogenous ZHX2 and ephrin-B intracellular fragment, embryonic day 15.5 (E15.5) fetal mouse cortices were lysed in radioimmunoprecipitation assay buffer. The lysate was homogenized by passing through a $27 \mathrm{G}$ needle and was cleared by centrifugation at $5000 \mathrm{~g}$ at $4^{\circ} \mathrm{C}$. The supernatant was incubated with GST-ephrin-B affinity beads for pull-down or precleared with Talon beads followed by incubation with His6-ZHX2 affinity beads for pull-down. After incubation and washing, the beads were boiled in SDS sample buffer.

Protein samples were run on a SDS-PAGE gel (concentration ranging from 7.5 to $15 \%$ ), then transferred to a polyvinylidene difluoride membrane using a semidry trans-blotter (Bio-Rad). The membrane was blocked for $1 \mathrm{~h}$ in $5 \%$ nonfat milk, then incubated with primary antibody overnight at $4^{\circ} \mathrm{C}$. Binding of primary antibody was detected using a horseradish peroxidase-conjugated secondary antibody and chemiluminescent substrate (Pierce).

Dissociated primary cell culture. Cerebral cortices of E11 mouse embryos were dissected in HBSS (Irvine Scientific). Cells were dissociated using a pipette. After centrifugation at $1000 \mathrm{rpm} 4^{\circ} \mathrm{C}$ for $5 \mathrm{~min}$, cells were washed twice with HBSS. Cell pellets were then resuspended in D-MEM/ F12 medium (Invitrogen) supplemented with B27 (Sigma; 1:50 v/v), penicillin $(100 \mathrm{U} / \mathrm{ml})$, and streptomycin $(100 \mu \mathrm{g} / \mathrm{ml})$, counted and plated in a 24 -well plate at the confluency of $2 \times 10^{5}$ cells/well onto polyornithine $\left(15 \mu \mathrm{g} / \mathrm{ml}\right.$ in $\left.\mathrm{H}_{2} \mathrm{O}\right)$ and fibronectin $(2 \mu \mathrm{g} / \mathrm{ml}$ in PBS)coated coverslips, and cultured at $37^{\circ} \mathrm{C}$. After initial plating $(24 \mathrm{~h})$, cells were transfected in triplicate with an expression plasmid of ZHX2-VP16 or a plasmid of a control protein (EGFP) using Fugene6 (Roche). For BrdU labeling, BrdU was added to the culture at a final concentration of $10 \mu \mathrm{M}$ for $16 \mathrm{~h}$ before fixation. After transfection $(48 \mathrm{~h})$, cells were fixed with $4 \%$ paraformaldehyde for immunocytochemistry. The dissociated cell assays were repeated independently three times. Averages of the experiments were presented in quantification. The acutely dissociated cells derived from electroporated brains were prepared as described in a previous study (Qiu et al., 2008).

RNA in situ hybridization, immunohistochemistry, and immunocytochemistry. RNA in situ, immunohistochemistry, and immunocytochemistry were done essentially as in Qiu et al. (2008). Fluorescent images were acquired using a Zeiss Upright 510 two-photon confocal microscope. Digital images were processed (brightness/contrast adjusted and color balanced) using ImagePro Plus software (Media Cybernetics).

Luciferase assay. Plasmid with firefly luciferase driven by 2XMH100-TK minimal promoter was cotransfected with cytomegalovirus- $\beta$ gal, along with GAL4, GAL4-ZHX2, ephrin-B1CD, or GAL4ZHX2-VP16, into HEK293 cells or cortical neural progenitors. The total amount of DNAs transfected was kept constant, substituting with empty expression plasmid as a control. After transfection ( $48 \mathrm{~h}$ ), cells were lysed in reporter lysis buffer (Promega) and measured for luciferase activity using luciferase assay system (Promega) by an LMAX2 luminometer (Molecular Devices). $\beta$-Galactosidase activity was used as an internal control for normalization of transfection/expression efficiency.

In utero electroporation. In utero electroporation was performed on E13.5 embryos as described previously (Qiu et al., 2008). Electroporated brains were dissected out $2 \mathrm{~h}$ after a pulse labeling of BrdU at E14.5 or at a later stage and cryoprotected for embedding. Consecutive sections ( 10 $\mu \mathrm{m}$ ) of an injected brain were collected for immunohistochemistry with specific antibodies. Electroporated cells were detected by direct visualization of EGFP expressed from the pEF-Ub-GFP expression plasmids. Sections representative of the center region of the electroporated cells were used for quantification. Quantification of the labeled green cells was analyzed using ImagePro Plus program (Media Cybernetics).

\section{Results}

\section{ZHX2 binds to the cytoplasmic domain of ephrin-B}

We performed further binding studies to confirm the interaction between ZHX2 and ephrin-B originally observed in a yeast two-hybrid screen. Fusion proteins between GST and various fragments of the cytoplasmic domain of ephrin-B1 or between a His6 tag and ZHX2 were constructed (Fig. 1A). C-terminal truncations of ephrin-B1 were made to reflect the various conserved regions between ephrin-B1 and ephrin-B2 (Fig. 1A) [ZHX2 was originally isolated from two-hybrid screen using ephrin-B2 cytoplasmic domain as a bait (Lu et al., 2001)]. Truncations of ZHX2 were made to reflect the predicted zinc finger domains (Fig. $1 A$ ). First, affinity beads bearing GST and ephrin-B1 fusions in comparable amounts were used to bind the full-length or an N-terminal fragment of ZHX2 expressed in HEK293 cells. The fusion protein between GST and the entire ephrin-B1 cytoplasmic domain (GSTephrin-B1CD) could interact with ZHX2 (Fig. 1B, lane 2). Amino acids 263-278 was a minimal sequence of the C-terminal deletion of ephrin-B1CD that could bind to ZHX2, but sequence 263-294 seemed to bind much stronger (Fig. $1 B$, lanes 5 and 6). We further tested the binding ability of sequence 278-294 and found that it displayed weak binding to ZHX2 (Fig. $1 B$, lane 7). These results suggest that the binding domain of ephrin-B1 is located within the N-terminal portion (263-294) of the ephrin-B1 cytoplasmic domain, a region adjacent to the transmembrane domain and conserved between ephrin-B1 and ephrin-B2. Truncations of ZHX2 found that an $\mathrm{N}$-terminal fragment of $\mathrm{ZHX} 2$ that precedes the two zinc finger domains could bind to GST-ephrin-B1CD as strongly as the full-length protein. Because the original ZHX2 cDNA isolated in yeast two-hybrid screen encoded amino acids 27-157 (Fig. 1A), the binding domain of ZHX2 is likely to be located to amino acids 27-77. Next, GST-ephrin-B1CD was tested for interaction with endogenous $\mathrm{ZHX} 2$, and we found that it could bind to ZHX2 derived from E15.5 mouse cortices (Fig. $1 C)$. Finally, a reciprocal experiment using affinity beads bearing His6-ZHX2 was tested for interaction with endogenous ephrin-B in the mouse cortices. We found that His6-Zhx2 could enrich an ephrin-B antibody-reactive peptide with a size similar to a recombinant ephrin-B1CD control expressed in HEK293 cells (Fig. 1 D). This peptide and a peptide of a smaller size (also detected in His6-ZHX2 pull-down) were likely the endogenous intracellular fragments generated from ephrin-B1 and ephrin-B2, both were expressed in the developing cortex (Stuckmann et al., 2001; Qiu et al., 2008). These binding studies confirm a specific interaction between ZHX2 and the intracellular fragment of ephrin-B.

We further looked at the subcellular localization of ZHX2 and 


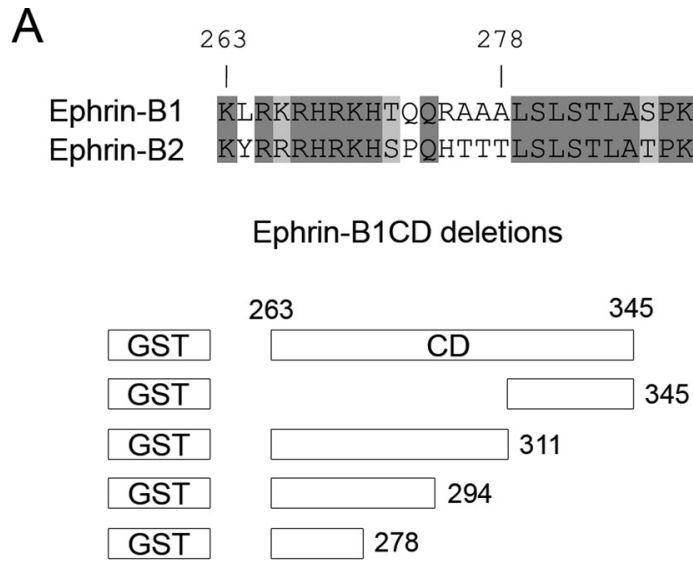

294

311

345

Ephrin-B1 KLRKRHRKHTQQRAAALSLSTLASPKGGSGTAGTEPSDII IPLRTTENNYCPHYEKVSGDYGHPVYIVQEMPPQSPANIYYKV

Ephrin-B2 KYRRRHRKHSPQHTTTLSLSTLATPKRGGNNNGSEPSDVIIPLRTADSVFCPHYEKVSGDYGHPVYIVQEMPPQSPANIYYKV
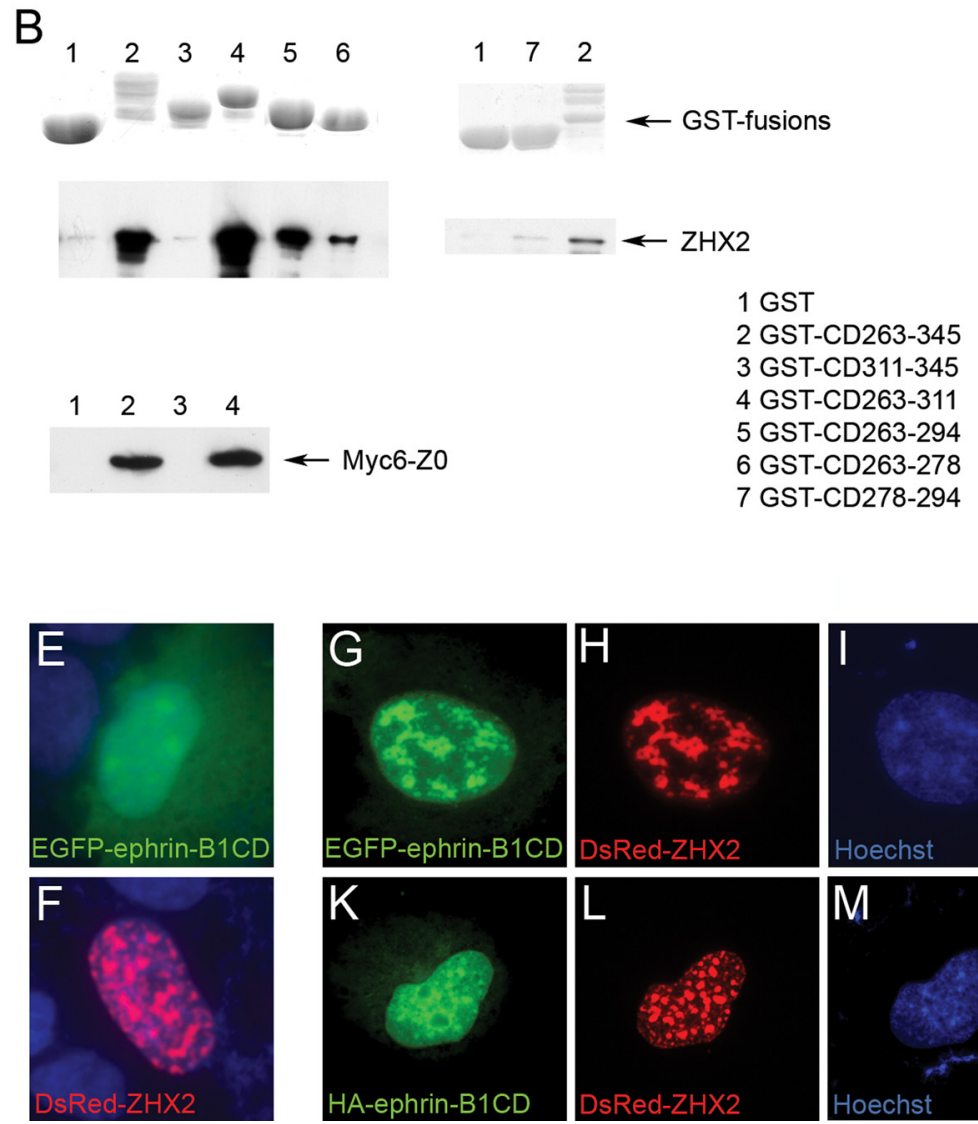
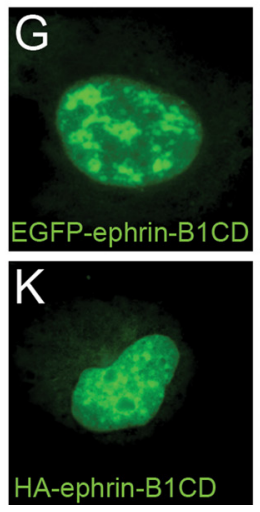
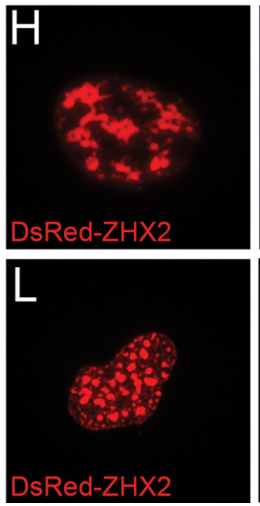

$\mathrm{ZHX2}$ deletion

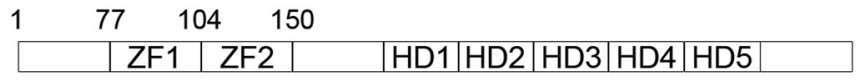

\section{\begin{tabular}{|l|l|}
\hline Myc6 & ZO \\
\hline
\end{tabular}}

\begin{tabular}{|l|l}
\hline$\quad$ Y2H clone \\
27
\end{tabular}

Figure 1. Interaction between ZHX2 and an intracellular fragment of ephrin-B. $A$, Sequence comparison between ephrin-B1 and ephrin-B2 cytoplasmic domains and schematic structures of ephrin-B1 cytoplasmic domain and ZHX2; $\boldsymbol{B}$, expression and binding of different GST-ephrin-B1 cytoplasmic domain fusion proteins to the full-length or a deletion of ZHX2 expressed in HEK293 cells. C, Binding of GST- ephrin-B1 cytoplasmic domain to endogenous ZHX2 in mouse cortical cell extract. $\boldsymbol{D}$, Binding of His6 6 -ZHX2 fusion protein to endogenous ephrin-B intracellular fragment in mouse cortical cell extract. $\boldsymbol{E}$, Subcellular localization of EGFP- ephrin-B1 cytoplasmic domain fusion protein in COS-7 cells; $\boldsymbol{F}$, nuclear localization of DsRed-ZHX2 fusion protein in COS-7 cells; $\mathbf{G}-\boldsymbol{N}$, colocalization of EGFP- or HA-tagged ephrin-B1 cytoplasmic domain fusion protein and DsRed-ZHX2 fusion protein in COS-7 cells.

ephrin-B1CD in transfected cells. GFP-tagged ephrin-B1CD was seen both in the cytoplasm and in the nucleus when expressed alone (Fig. 1E). DsRed-tagged ZHX2 (DsRed-ZHX2) was expressed restrictedly in the nucleus (Fig. $1 F$ ). When GFP-ephrinB1CD was coexpressed with DsRed-ZHX2, it colocalized with DsRed-ZHX2 in the nucleus (Fig. $1 G-J$ ). Colocalization of ephrin-B1CD and ZHX2 in the nucleus was also seen by immunocytochemistry when a smaller tag (HA) was used to mark ephrin-B1CD (Fig. $1 \mathrm{~K}-\mathrm{N}$ ). In the latter case, proteasomal inhibitor MG-132 was present in the culture medium to prevent deg- radation of the HA-tagged ephrin-B1CD. These results suggest that ZHX2 can recruit ephrin-B1CD in the nucleus.

\section{$\mathrm{ZHX} 2$ is expressed in cortical neural progenitors during} cortical neurogenesis

We looked at the expression pattern of ZHX2 in the developing mouse brain compared with known patterns of expression of ephrin-B. We found that the mRNA of ZHX2 was selectively expressed in the $\mathrm{VZ}$ of the cortex (Fig. 2A), similar to what was observed for ephrin-B1 (Lu et al., 2001). Immunohistochemistry 



Figure 2. Expression of ZHX2 in cortical neural progenitor cells. A, RNA in situ hybridization of ZHX2 in E15.5 embryonic cortex. ctx, Cerebral cortex; ge, ganglion eminence. $\boldsymbol{B}$, Immunohistochemistry of ZHX2 in E12.5, E14.5, and E15.5 cortices. C, Coimmunostaining of ZHX2 with nestin in neural progenitors. D, Costaining of ZHX2 with PAX6. E, Costaining of ZHX2 with incorporated BrdU. Scale bars, $50 \mu \mathrm{m}$.

showed that ZHX2 protein expression could be detected in the $\mathrm{VZ}$ and subventricular zone (SVZ) of the cortex during various stages of cortical neurogenesis (Fig. $2 B$ ) and correlated with the expression of nestin (Fig. 2C), suggesting that ZHX2 is expressed in neural progenitor cells. Costaining of ZHX2 with PAX6 showed substantial overlap of the two proteins (Fig. 2D), indicating that ZHX2 is mainly expressed by radial glia progenitors. However, we were unable to determine whether ZHX2 was also expressed by basal progenitors (Haubensak et al., 2004; Miyata et al., 2004; Noctor et al., 2004), attributable to incompatibility of antibodies of ZHX2 and Tbr2 (Englund et al., 2005). Some of the ZHX2-expressing cells were positive for incorporated BrdU (Fig. $2 E$ ), further suggesting that ZHX2 is expressed in neural progenitor cells. This expression pattern suggests that ZHX2 is likely a biologically relevant interactor with ephrin-B intracellular fragment and that ZHX2 may have a role in the regulation of neural progenitor maintenance similar to ephrin-B.
ZHX2 is a transcriptional repressor, and converting it into a transcriptional activator causes differentiation of neural progenitor cells

Consistent with previous reports (Kawata et al., 2003a,b; Perincheri et al., 2005; Liu et al., 2006), we found that GAL4-ZHX2 (a fusion protein between the DNA-binding domain of GAL4 and ZHX2) could inhibit basal transcription of a GAL4 reporter in HEK293 cells, as well as in cortical neural progenitor cells derived from early embryonic cortices (Fig. 3A). Interestingly, coexpression of ephrinB1CD resulted in a stronger repression of transcription by GAL4ZHX2 without affecting the expression level of GAL4-ZHX2 protein (Fig. 3A) (data not shown). As basal activity of transcription was generally low in cells, we further looked at this effect of ephrinB1CD using a fusion protein between GAL4-ZHX2 and the transcriptional activation domain of VP16. GAL4-ZHX2-VP16 fusion protein could activate transcription $>30$-fold over the basal level (data not shown). When ephrin-B1CD was coexpressed in HEK 
cells, it could inhibit GAL4-ZHX2-VP16activated transcription in a dose-dependent manner but further enhanced the activated transcription induced by a control protein GAL4-VP16 (Fig. 3B). Similarly, coexpression of ephrin-B1CD with GAL4-ZHX2VP16 could suppress GAL4-ZHX2-VP16 activation in cortical neural progenitor cells (Fig. 3B).

The action of ZHX2 as a transcription regulator suggests that $\mathrm{ZHX} 2$ is likely to exert its function by means of controlling, or more specifically inhibiting, the expression of target genes in cortical neural progenitor cells. To probe the possible function of ZHX2, we sought to express ZHX2-VP16 fusion protein in cortical neural progenitors. GAL4-ZHX2-VP16 and GAL4-ZHX2 could compete with each other in transcription (data not shown); we, therefore, reasoned that expression of ZHX2-VP16 would compete for the endogenous ZHX proteins (either homodimerized or heterodimerized proteins) and activate the ZHX2 target genes, thus disrupting the normal repressor function of ZHX2. We first introduced ZHX2VP16 into isolated cortical neural progenitors cultured in vitro and tested its possible effect on cell fate change in the affected cells. Expression of ZHX2-VP16 (Fig. 3C) caused a reduction in the number of nestin-positive (ZHX2-VP16, 75.2\% vs EGFP, 93.6\%) and BrdU-incorporating cells (ZHX2-VP16, 41.5\% vs EGFP, $72.4 \%$ ) but an increase in the population of DCX-positive cells (ZHX2-VP16, $20.8 \%$ vs EGFP, $9.6 \%$ ). The lower percentage of BrdU-positive cells detected in the culture, compared with the nestin-positive cells, might be attributable to an insufficient antigen exposure. Overall, these results suggest that interfering with $\mathrm{ZHX} 2$ function could induce neuronal differentiation, thereby reducing the number of neural progenitors.

Expression of ZHX2-VP16 in the cortex causes differentiation of neural progenitor cells

To further examine the function of $\mathrm{ZHX} 2$ in neural progenitors, we used in utero electroporation-mediated DNA delivery (Fukuchi-Shimogori and Grove, 2001; Bai et al., 2003; Elias et al., 2007; Mizutani et al., 2007; Wang et al., 2007) to express ZHX2-VP16 in the developing cortex. Analyses of cortical neural progenitors at E14.5 (electroporation at E13.5 and analysis of brain at E14.5) (Fig. 4A) showed that expression of ZHX2VP16 led to a decrease in the number of cells positive for BrdU incorporation (ZHX2-VP16, 17.7\% vs EGFP, 23.3\%), phosphohistone $\mathrm{H} 3$ (ZHX2-VP16, 4.1\% vs EGFP, 7.2\%), or Tbr2 Student's $t$ test. $p<0.001$.
A

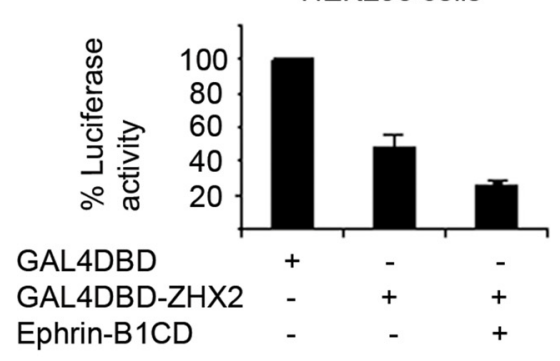

B
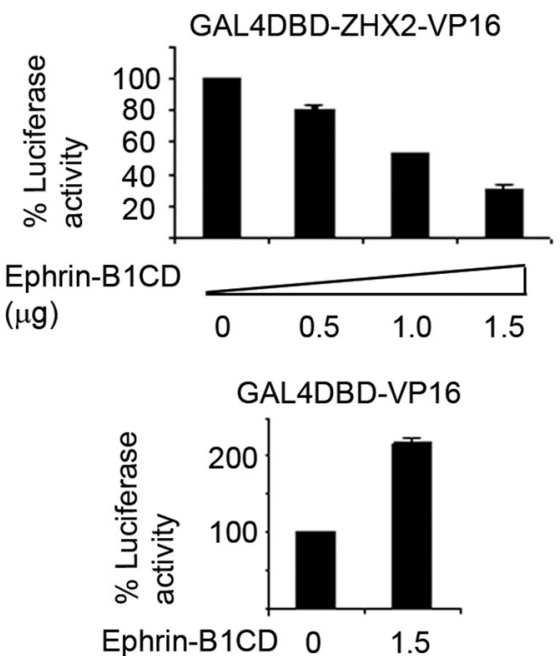

$(\mu \mathrm{g})$
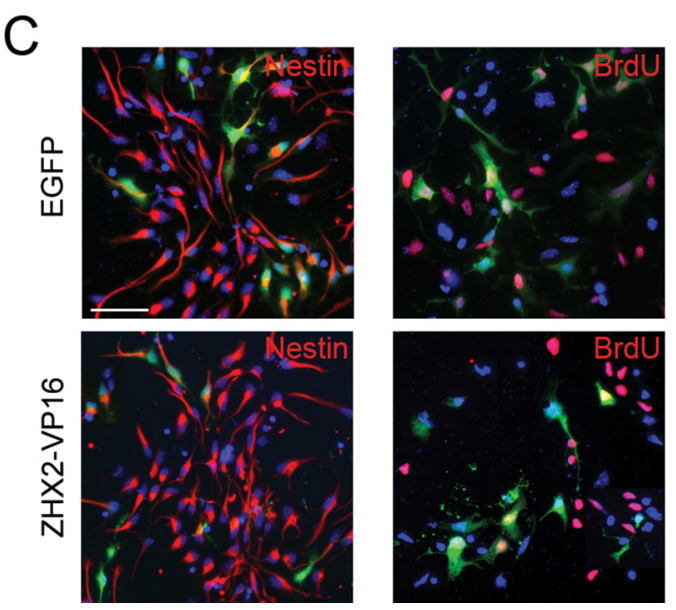

Cortical neural progenitors

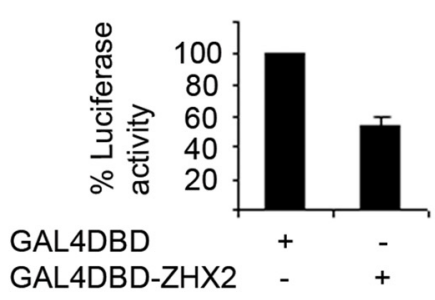

Cortical neural progenitors GAL4DBD-ZHX2-VP16

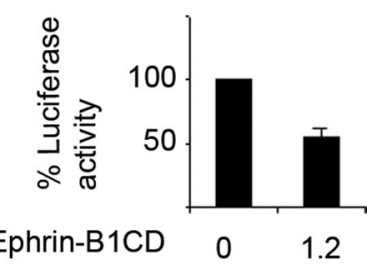

$(\mu \mathrm{g})$
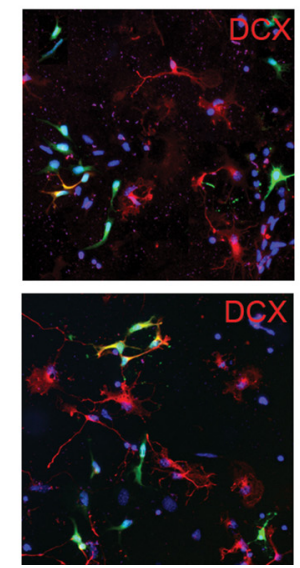

Figure 3. Transcriptional repression by ZHX2 and neuronal differentiation of cortical neural progenitors induced by ZHX2VP16. A, Luciferase assay in transfected HEK293 cells and cortical progenitor cells. Luciferase activity of control cells containing GAL4 DNA-binding domain (GAL4DBD) alone was represented as 100\%. B, Effect of ephrin-B1 intracellular domain on transcriptional activity of GAL4-ZHX2-VP16 in HEK293 cells or cortical progenitor cells. C, Expression of control EGFP or ZHX2-VP16 in neural progenitor cells derived from E11 cortices. Cells were fixed for immunocytochemistry of nestin, doublecortin, or incorporated BrdU $48 \mathrm{~h}$ after transfection. Percentage of green cells positive for each marker was determined. Nestin, EGFP: $93.6 \%$ (165 of 176), ZHX2-VP16: 75.2\% (139 of 185); BrdU, EGFP: 72.4\% (135 of 187), ZHX2-VP16: 41.5\% (59 of 142); doublecortin, EGFP: 9.6\% (21 of 219), ZHX2-VP16: 20.8\% (39 of 186). Scale bar, $50 \mu \mathrm{m}$. Error bars show SEM. Statistical analysis was done using

(ZHX2-VP16, 2.6\% vs EGFP, 6.5\%). Analyses of the electroporated mouse cortices at E15.5 (electroporation at E13.5 and analysis of brain at E15.5) showed that ZHX2-VP16 caused cells to move away from the apical part of the ventricular zone, showing 

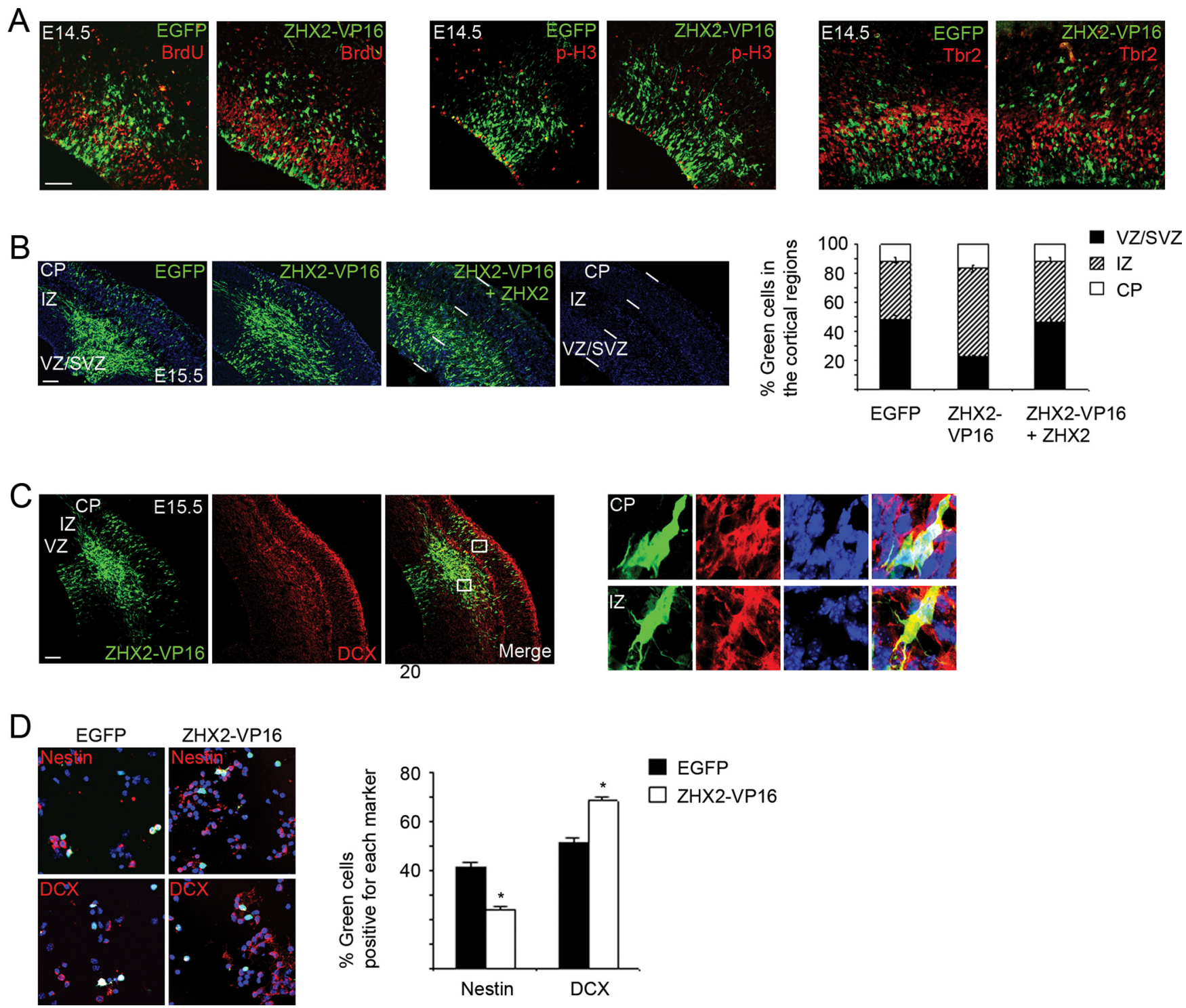

Figure 4. Interfering with ZHX2 function in the cerebral cortex. A, Embryonic brains were electroporated with expression plasmids at E13.5 and sectioned at E14.5 for immunohistochemistry of pulse-labeled BrdU, phosphohistone $\mathrm{H3}$ (p-H3), or Tbr2. More than seven brains were analyzed for each tested condition. Percentages of BrdU-positive green cells, apically localized p-H3-positive green cells, and Tbr2-positive green cells were as follows: BrdU, EGFP: 23.3\%, ZHX2-VP16: 17.7\%; pH-3, EGFP: 7.2\%, ZHX2-VP16: 4.1\%; Tbr2, EGFP: 6.5\%, ZHX2-VP16: 2.6\%. B, Electroporated brains were analyzed at E15.5. Seven or more brains were analyzed for each electroporated plasmid. The distributions (percentage) of green cells in VZ/SVZ, IZ, and CP were determined. The cortical areas (VZ/SVZ, IZ, and (P) were delineated by Hoechst staining that revealed demarcation of cell packing. EGFP: 48.1, 40.6, 11.3\%; ZHX2-VP16:22.7, 60.7, 16.8\%; ZHX2-VP16 plus ZHX2: 46.7, 41.2, 12.1\%. C, ZHX2-VP16-expressing cells in the CP or IZ were positive for neuronal marker DCX. D, Immunocytochemistry on cellular markers in acutely dissociated cells obtained from fluorescent (EGFP) regions of the electroporated cortices (E15.5). Nestin, EGFP: 41\% (68 of 166); ZHX2-VP16: 23.5\% (42 of 179); DCX, EGFP: $50.9 \%$ (56 of 110); ZHX2-VP16: $68.2 \%$ (118 of 173). Scale bars, 50 $\mu \mathrm{m}$. Error bars show SEM. Statistical analysis was done using Student's $t$ test. ${ }^{*} p<0.005$.

proportionally more cells in the intermediate zone (IZ) and the cortical plate $(\mathrm{CP})$ comparing to the control cells expressing EGFP (Fig. 4B, C). Coexpression of ZHX2 could suppress this effect of ZHX2-VP16 (Fig. 4B), suggesting that ZHX2-VP16 acts through blocking the function of endogenous ZHX2 protein. Immunohistochemistry on ZHX2-VP16-expressing cells in the CP and IZ revealed that these cells were doublecortin positive (Fig. $4 C$ ), suggesting that the cells leaving VZ and entering IZ and CP are mostly differentiated cells. Consistent with this observation, cortical cells acutely dissociated from the electroporated cortices at E15.5 showed that expression of ZHX2-VP16 in the cortex resulted in an increase of doublecortin-positive cells and a decrease of nestin-positive cells (Fig. 4D). Together, these results suggest that interfering with ZHX2 function in the cortex leads to neuronal differentiation of neural progenitor cells, similar to what was observed in cortical neural progenitors cultured in vitro.

\section{Expression of ZHX2 in the cortex disrupts normal} differentiation of neural progenitor cells

We further tested the effect of overexpression of ZHX2 in the cortex. If ZHX2 acts to prevent differentiation as suggested by the data of ZHX2-VP16, expression of ZHX2 in neural progenitor cells might be expected to inhibit differentiation. We, therefore, looked at the experimental brains at E17.5, $4 \mathrm{~d}$ after electroporation, when most of the control cells (e.g., expressing EGFP) should have differentiated and migrated outwards into the cortical plate (Qiu et al., 2008) (Fig. $5 A$ ). We found that expression of ZHX2 in the cortex caused proportionally more cells to stay behind in the VZ without moving into 
A
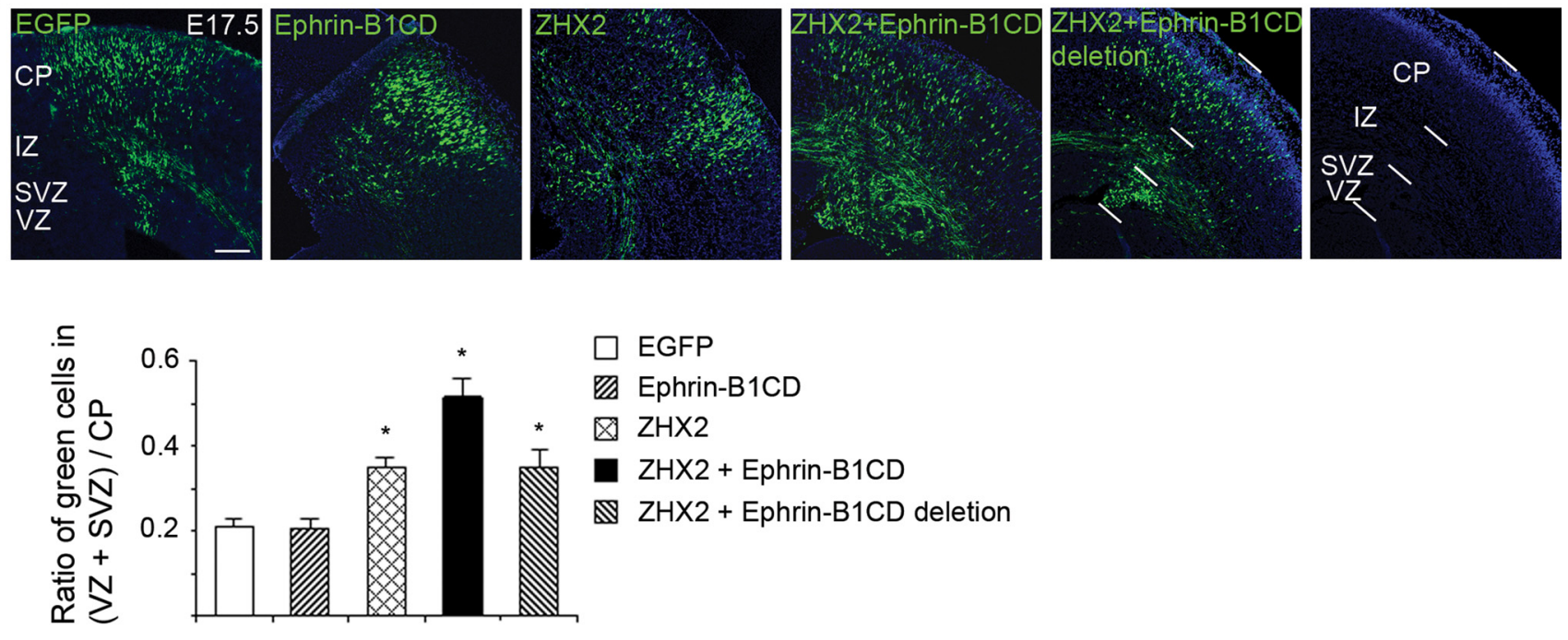

\author{
EGFP \\ Ephrin-B1CD \\ Z $\mathrm{HXX} 2$ \\ $\mathrm{ZHX} 2+$ Ephrin-B1CD \\ $\mathbb{Z}$ ZHX2 + Ephrin-B1CD deletion
}
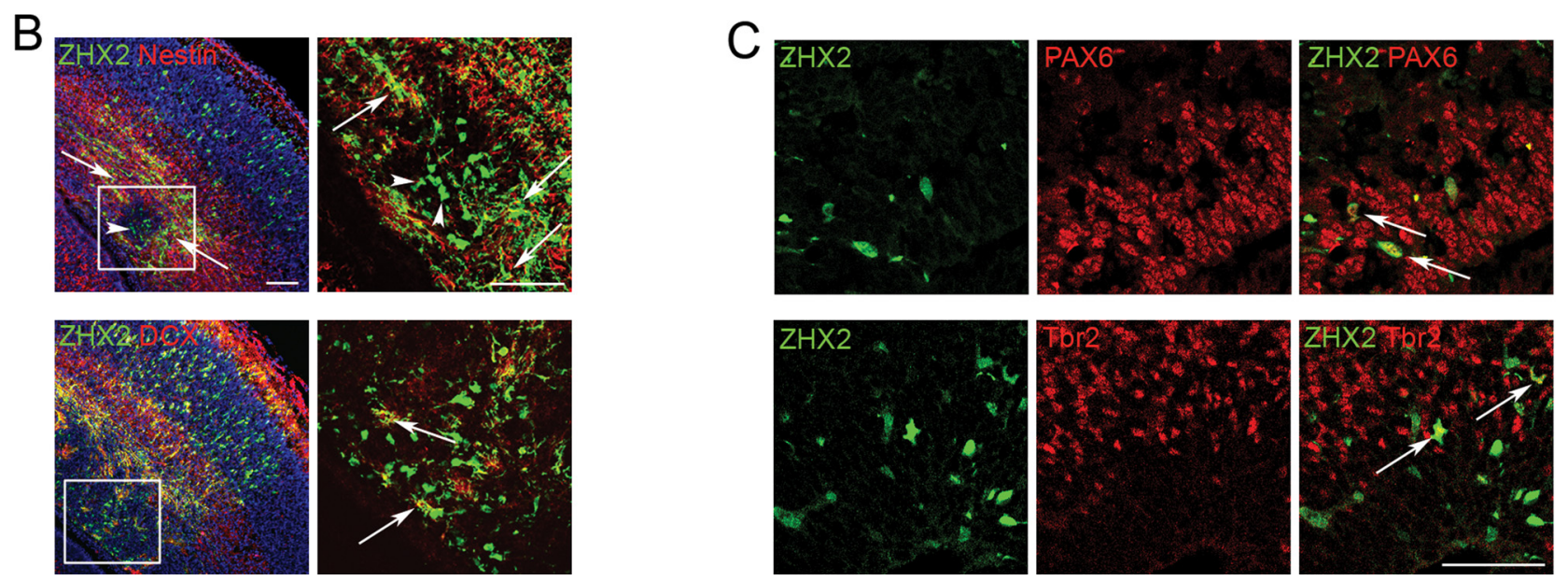

Figure 5. Expression of ZHX2 and ephrin-B1 intracellular domain in the cerebral cortex. $A$, Expression of $Z H X 2$ and ephrin-B1 intracellular fragment caused proportionally more cells to stay in the VZ and SVZ area. Ratios of green cells in the VZ/SVZ area versus green cells in the CP were quantified. EGFP, 0.21 ; ephrin-B1CD, $0.2 ; Z$ ZHX2, $0.35 ; Z$ ZHX2 plus ephrin-B1CD, $0.51 ; Z$ ZHX2 plus ephrin-B1CD deletion, 0.36 . Error bars show SEM. Statistical analysis was done using Student's $t$ test. ${ }^{*} p<0.01$. Six or more brains were analyzed for each electroporated plasmid. $\boldsymbol{B}$, Cell marker staining found that among the cells retained in the VZ/SVZ, there was a mixed population of nestin-positive cells $(\sim 84 \%)$ and doublecortin-positive cells ( $\sim 19 \%)$ (indicated by arrows). In some cases, expression of ZHX2 led to a disruption of radial organization in the neighboring area (indicated by arrowheads). C, ZHX2-expressing cells in the VZ/SVZ area contain both PAX6-positive and Tbr2-positive progenitors. Scale bar, $100 \mu \mathrm{m}$.

the outer cortical layers (Fig. 5A). The ratio of green cells in $\mathrm{VZ}$ and SVZ versus CP was used to document this effect. The IZ area in many of the E17.5 brain sections displayed strong labeling of axons which made the quantification of cell numbers difficult and was, therefore, not included in the analyses. Interestingly, coexpression of the ephrin-B1 cytoplasmic domain with ZHX2 could enhance this effect of ZHX2, whereas a deletion mutant of ephrin-B1 cytoplasmic domain which lacks the ZHX2-binding domain failed to do so (Fig. $5 A$ ). We next looked at the cell marker expression on these cells. Immunohistochemistry on nestin found that many ZHX2expressing cells in the VZ/SVZ were positive for nestin but some were negative (Fig. $5 B$ ). In several cases, we observed that the area surrounding ZHX2-expressing but nestin-negative cells displayed a disruption of the normal radial pattern of nestin staining (Fig. $5 B$, arrowhead). As we did not observe elevated cell apoptosis in this area (data not shown), the loss of nestin expression in the neighboring cells was likely attributable to a noncell autonomous effect of ZHX2 expression. Immunohistochemistry on doublecortin also revealed that some ZHX2-expressing cells were positive for this early neuron marker, and a few of them were located in the region of the VZ close to the ventricle, where doublecortin-expressing cells are rarely detected under normal circumstances. It appeared that differentiation in these ZHX2-expressing cells were not affected, but they did not complete the normal differentiation process of moving into the cortical plate, perhaps being retained in the VZ because of a disruption of the radial scaffold as observed in the nestin staining. A further look at the neural progenitor markers showed that the ZHX2-expressing cells in the VZ/SVZ area were positive for PAX6 and Tbr2 (Fig. 5C), suggesting that they contain both apical and basal neural progenitors. Together, these results suggest that overexpression of ZHX2 disrupts the normal differentiation program of cortical neural progenitors via inhibition of differentiation in some cells or disruption of the radial organization in others.

\section{Discussion}

Our results identify $\mathrm{ZHX} 2$ as a novel regulator in the maintenance of neural progenitor cells. This is supported by the following observations. First, ZHX2 is seen specifically expressed in 
neural progenitor cells during cortical neurogenesis, consistent with a role in neural progenitor maintenance. Second, ZHX2 is found to be able to bind to the intracellular fragment of ephrin-B, which is also selectively present in cortical neural progenitors and has a function in supporting neural progenitor maintenance (Qiu et al., 2008). Coexpression of ZHX2 and ephrin-B1 intracellular fragment could potentiate the transcriptional repressor activity of ZHX2 as well as enhance the effect of overexpression of ZHX2 in the cortex, suggesting that the two proteins may function in coordination in neural progenitors. Furthermore, interfering with ZHX2 function by the expression of a mutant ZHX2 protein, ZHX2-VP16, causes neuronal differentiation both in isolated neural progenitors and in the developing cortex, whereas overexpression of ZHX2 appears to partially inhibit the normal differentiation.

As observed in other cell types (Kawata et al., 2003a,b; Perincheri et al., 2005; Liu et al., 2006), we found that ZHX2 could behave as a transcriptional repressor in cortical neural progenitor cells. This suggests that the function of ZHX2 in neural progenitor cell maintenance is likely mediated by inhibiting the expression of a subset of genes. This role of ZHX2 may in part be achieved by forming heterodimers with ZHX1 and ZHX3 (Kawata et al., 2003b), which are also expressed in the developing cortex (data not shown). It is likely that some of the downstream genes regulated by ZHX2 homodimers or heterodimers are neurogenic. These ZHX2 target genes can induce neuronal differentiation when ZHX2 repression is released, when the normal expression of $\mathrm{ZHX} 2$ is downregulated during the onset of neurogenesis or as in the case of expression of ZHX2-VP16, which would be expected to compete for the function of homodimerized or heterodimerized ZHX2 and reverse the transcriptional repression to activation. Future identification of the target genes of ZHX2 will help better understand the molecular mechanism of $\mathrm{ZHX} 2$ function in neural progenitor cells.

How the ephrin-B1 intracellular domain modulates the transcriptional activity of $\mathrm{ZHX} 2$ is not clear. The entire ephrin-B1 cytoplasmic domain is relatively short in length and does not contain any obvious enzymatic activity that may be used to modulate binding proteins. One possibility is that ephrin-B1 intracellular domain may directly or indirectly interact with a component of the basic transcriptional machinery and that binding with ZHX2 can bring this component into the transcriptional complex of ZHX2, thereby modulating the activity of ZHX2. In addition, the binding domain of ephrin-B for interaction with ZHX2 was mapped to a region adjacent to the transmembrane domain, which is not involved in binding to other ephrin-B-interacting proteins, such as PDZ-RGS3 (Lu et al., 2001), Grb4 (Cowan and Henkemeyer, 2001), or STAT3 (Bong et al., 2007). This suggests that binding of the intracellular fragment of ephrin-B to each individual interactor may not be exclusive in the cell. Therefore, it is also likely that the ephrin-B intracellular domain may bring additional cofactor(s) into the complex with ZHX2 and together they modulate the activity of ZHX2. It will be interesting to further look into the presence of a possible multicomplex of ephrin-B and the interacting proteins and test the function of such a complex in the regulation of neural progenitor cells.

In the reverse signaling of the ephrin-Eph-mediated cellcell communication, the cytoplasmic domain of ephrin-B transduces a signal through either phosphorylationdependent or phosphorylation-independent interaction with signal transducer proteins, including cytosolic proteins Grb4/
Nck2 (Cowan and Henkemeyer, 2001), PTP-BL (Lin et al., 1999; Palmer et al., 2002), and PDZ-RGS3 (Lu et al., 2001; Qiu et al., 2008). Ephrin-B was also reported to elicit a nuclear signaling via phosphorylation-dependent interaction through JAK/STAT3 pathway (Bong et al., 2007). Our observation of a direct interaction between ZHX2 and the cytosolic fragment of ephrin-B suggests a potential novel nuclear mechanism of ephrin-B, often used by type I transmembrane proteins, such as Notch and amyloid precursor protein (Selkoe and Kopan, 2003). It is conceivable that in this mechanism ephrin-B cytoplasmic domain translocates into the nucleus, binds to ZHX2, and modulates $\mathrm{ZHX} 2$ activity in transcriptional control. In support for this idea, we have observed that the cytoplasmic domain of ephrin-B1 is recruited by ZHX2 in the nucleus and can enhance the transcriptional repressor activity of ZHX2. Coexpression of an ephrin-B1 intracellular fragment in the cortex was also found to augment a blockade of differentiation induced by ZHX2. Identification of target promoters of ZHX2 will provide a platform for further characterization of the interaction between $\mathrm{ZHX} 2$ and ephrin-B in regulating target gene transcription. With respect to ephrin-B function, our observed interaction between ZHX2 and ephrin-B suggests that ephrin-B may use multiple mechanisms in neural progenitor maintenance including a PDZ-RGS3-mediated pathway (Qiu et al., 2008) and a ZHX protein-mediated mechanism.

\section{References}

Arvanitis D, Davy A (2008) Eph/ephrin signaling: networks. Genes Dev 22:416-429.

Bai J, Ramos RL, Ackman JB, Thomas AM, Lee RV, LoTurco JJ (2003) RNAi reveals doublecortin is required for radial migration in rat neocortex. Nat Neurosci 6:1277-1283.

Barthelemy I, Carramolino L, Gutiérrez J, Barbero JL, Márquez G, Zaballos A (1996) zhx-1: a novel mouse homeodomain protein containing two zincfingers and five homeodomains. Biochem Biophys Res Commun 224:870-876.

Bong YS, Lee HS, Carim-Todd L, Mood K, Nishanian TG, Tessarollo L, Daar IO (2007) ephrinB1 signals from the cell surface to the nucleus by recruitment of STAT3. Proc Natl Acad Sci U S A 104:17305-17310.

Bystron I, Blakemore C, Rakic P (2008) Development of the human cerebral cortex: Boulder Committee revisited. Nat Rev Neurosci 9:110-122.

Caviness VS, Bhide PG, Nowakowski RS (2008) Histogenetic processes leading to the laminated neocortex: migration is only a part of the story. Dev Neurosci 30:82-95.

Cowan CA, Henkemeyer M (2001) The SH2/SH3 adaptor Grb4 transduces B-ephrin reverse signals. Nature 413:174-179.

Cowan CA, Henkemeyer M (2002) Ephrins in reverse, park and drive. Trends Cell Biol 12:339-346.

Davy A, Soriano P (2005) Ephrin signaling in vivo: look both ways. Dev Dyn 232:1-10

Drescher U (2002) Eph family functions from an evolutionary perspective. Curr Opin Genet Dev 12:397-402.

Egea J, Klein R (2007) Bidirectional Eph-ephrin signaling during axon guidance. Trends Cell Biol 17:230-238.

Elias LA, Wang DD, Kriegstein AR (2007) Gap junction adhesion is necessary for radial migration in the neocortex. Nature 448:901-907.

Englund C, Fink A, Lau C, Pham D, Daza RA, Bulfone A, Kowalczyk T, Hevner RF (2005) Pax6, Tbr2, and Tbr1 are expressed sequentially by radial glia, intermediate progenitor cells, and postmitotic neurons in developing neocortex. J Neurosci 25:247-251.

Fishell G, Kriegstein AR (2003) Neurons from radial glia: the consequences of asymmetric inheritance. Curr Opin Neurobiol 13:34-41.

Flanagan JG, Vanderhaeghen P (1998) The ephrins and Eph receptors in neural development. Annu Rev Neurosci 21:309-345.

Fortini ME (2002) Gamma-secretase-mediated proteolysis in cell-surfacereceptor signalling. Nat Rev Mol Cell Biol 3:673-684.

Fukuchi-Shimogori T, Grove EA (2001) Neocortex patterning by the secreted signaling molecule FGF8. Science 294:1071-1074.

Georgakopoulos A, Litterst C, Ghersi E, Baki L, Xu C, Serban G, Robakis NK 
(2006) Metalloproteinase/Presenilin1 processing of ephrinB regulates EphB-induced Src phosphorylation and signaling. EMBO J 25:1242-1252.

Götz M, Huttner WB (2005) The cell biology of neurogenesis. Nat Rev Mol Cell Biol 6:777-788.

Gupta A, Tsai LH, Wynshaw-Boris A (2002) Life is a journey: a genetic look at neocortical development. Nat Rev Genet 3:342-355.

Haubensak W, Attardo A, Denk W, Huttner WB (2004) Neurons arise in the basal neuroepithelium of the early mammalian telencephalon: a major site of neurogenesis. Proc Natl Acad Sci U S A 101:3196-3201.

Kawata H, Yamada K, Shou Z, Mizutani T, Yazawa T, Yoshino M, Sekiguchi T, Kajitani T, Miyamoto K (2003a) Zinc-fingers and homeoboxes (ZHX) 2, a novel member of the ZHX family, functions as a transcriptional repressor. Biochem J 373:747-757.

Kawata H, Yamada K, Shou Z, Mizutani T, Miyamoto K (2003b) The mouse zinc-fingers and homeoboxes (ZHX) family; ZHX2 forms a heterodimer with ZHX3. Gene 323:133-140.

Lin D, Gish GD, Songyang Z, Pawson T (1999) The carboxyl terminus of B class ephrins constitutes a PDZ domain binding motif. J Biol Chem 274:3726-3733.

Liu G, Clement LC, Kanwar YS, Avila-Casado C, Chugh SS (2006) ZHX proteins regulate podocyte gene expression during the development of nephrotic syndrome. J Biol Chem 281:39681-39692.

Lu Q, Sun EE, Klein RS, Flanagan JG (2001) Ephrin-B reverse signaling is mediated by a novel PDZ-RGS protein and selectively inhibits G proteincoupled chemoattraction. Cell 105:69-79.

McConnell SK (1995) Constructing the cerebral cortex: neurogenesis and fate determination. Neuron 15:761-768.

Miyata T, Kawaguchi A, Saito K, Kawano M, Muto T, Ogawa M (2004) Asymmetric production of surface-dividing and non-surface-dividing cortical progenitor cells. Development 131:3133-3145.

Mizutani K, Yoon K, Dang L, Tokunaga A, Gaiano N (2007) Differential Notch signalling distinguishes neural stem cells from intermediate progenitors. Nature 449:351-355.

Molyneaux BJ, Arlotta P, Menezes JR, Macklis JD (2007) Neuronal subtype specification in the cerebral cortex. Nat Rev Neurosci 8:427-437.

Monuki ES, Walsh CA (2001) Mechanisms of cerebral cortical patterning in mice and humans. Nat Neurosci 4 [Suppl]:1199-1206.

Noctor SC, Martínez-Cerdeño V, Ivic L, Kriegstein AR (2004) Cortical neu- rons arise in symmetric and asymmetric division zones and migrate through specific phases. Nat Neurosci 7:136-144.

Noctor SC, Martinez-Cerdeno V, Kriegstein AR (2007) Neural stem and progenitor cells in cortical development. Novartis Found Symp 288:5973; discussion 73-78, 96-98.

Palmer A, Zimmer M, Erdmann KS, Eulenburg V, Porthin A, Heumann R, Deutsch U, Klein R (2002) EphrinB phosphorylation and reverse signaling: regulation by Src kinases and PTP-BL phosphatase. Mol Cell 9:725-737.

Pasquale EB (2008) Eph-ephrin bidirectional signaling in physiology and disease. Cell 133:38-52.

Perincheri S, Dingle RW, Peterson ML, Spear BT (2005) Hereditary persistence of alpha-fetoprotein and $\mathrm{H} 19$ expression in liver of BALB/cJ mice is due to a retrovirus insertion in the Zhx2 gene. Proc Natl Acad Sci U S A 102:396-401.

Poliakov A, Cotrina M, Wilkinson DG (2004) Diverse roles of eph receptors and ephrins in the regulation of cell migration and tissue assembly. Dev Cell 7:465-480.

Pontious A, Kowalczyk T, Englund C, Hevner RF (2008) Role of intermediate progenitor cells in cerebral cortex development. Dev Neurosci 30:24-32.

Qiu R, Wang X, Davy A, Wu C, Murai K, Zhang H, Flanagan JG, Soriano P, Lu Q (2008) Regulation of neural progenitor cell state by ephrin-B. J Cell Biol 181:973-983.

Rash BG, Grove EA (2006) Area and layer patterning in the developing cerebral cortex. Curr Opin Neurobiol 16:25-34.

Selkoe D, Kopan R (2003) Notch and Presenilin: regulated intramembrane proteolysis links development and degeneration. Annu Rev Neurosci 26:565-597.

Stuckmann I, Weigmann A, Shevchenko A, Mann M, Huttner WB (2001) Ephrin B1 is expressed on neuroepithelial cells in correlation with neocortical neurogenesis. J Neurosci 21:2726-2737.

Temple S (2001) The development of neural stem cells. Nature 414:112-117.

Tomita T, Tanaka S, Morohashi Y, Iwatsubo T (2006) Presenilin-dependent intramembrane cleavage of ephrin-B1. Mol Neurodegener 1:2.

Wang X, Qiu R, Tsark W, Lu Q (2007) Rapid promoter analysis in developing mouse brain and genetic labeling of young neurons by doublecortinDsRed-express. J Neurosci Res 85:3567-3573. 